\title{
Assessing the Presence and Position of Carotid Plaque Improves Risk Stratification for Cardiovascular Disease Prediction Among Patients With Hypertension
}

Hui-Juan Zuo ( $\nabla$ huijuanzuo@sina.com )

Beijing Anzhen Hospital

Xian-Tao Song

Beijing Anzhen Hospital

Jin-Wen Wang

Beijing Anzhen Hospital

Hong-Xia Yang

Beijing Anzhen Hospital

Jie Lin

Beijing Anzhen Hospital

\section{Research Article}

Keywords: Ischemic Cardiovascular Disease, Hypertension, Carotid Plaque, Risk Stratification

Posted Date: June 4th, 2021

DOI: https://doi.org/10.21203/rs.3.rs-574219/v1

License: (c) (i) This work is licensed under a Creative Commons Attribution 4.0 International License.

Read Full License 


\section{Abstract}

Background: Ischemic cardiovascular disease (ISCVD) is a massive public health problem. ISCVD risk prediction models based on traditional risk factors as predictors is limited. Carotid atherosclerosis plays a fundamental value in the occurrence of ISCVD. The aim of this study was to evaluate the value of risk stratification plus carotid plaque improving the prediction of ISCVD.

Methods: Between June 2016 and June 2017, 3998 subjects with hypertension were prospectively recruited and completed traditional risk factors survey and carotid ultrasound measurements in Anzhen Hospital, Beijing, China.

Results: A total of 2010 (50.3\%) subjects were detected carotid plaque. Among patients free from ISCVD $(n=3479)$, there were 884 patients $(25.4 \%)$ at high risk for ISCVD, and $868(25.0 \%), 1727(49.6 \%)$ was classified as intermediate risk or low risk according to Chinese cardiovascular risk score chart. The detected rate of carotid plaque was $64.7 \%, 53.7 \%$, and $38.5 \%$ among patients at high risk to low risk, respectively. Carotid plaques and risk stratification alone or in combination were significantly associated with ischemic stroke, and negatively correlated with coronary heart disease (all $P>0.05$ ). Adding carotid plaque to risk stratification, the ischemic stroke prevalence increased from $5.3 \%$ to $9.1 \%$ in the low-risk group $(P=0.001), 5.4 \%$ to $12.3 \%$ in the intermediate-risk group $(P<0.001)$ and $8.2 \%$ to $14.4 \%$ than in the high-risk group $(P=0.004)$. Intermediate risk plus carotid plaque $(443 / 3998)$ were reclassified to a new high-risk group, high risk only (749/3998) and low risk plus carotid plaque (353/3998) were reclassified to a new intermediate risk group; and intermediate risk only (553/3998) were reclassified to a new low risk group. According to the reclassification, there were 1635 subjects (40.9\%) at high risk, and 1102 (27.6\%), 1261 (31.5\%) was classified as intermediate risk or low risk.

Conclusions: Carotid plaque has an important position as it plus risk stratification may improve the risk assessment of ischemic stroke and have resulted in reclassification.

\section{Background}

Cardiovascular disease (CVD) is a massive public health problem. Hypertension is a leading risk factor for CVD [1], and has reached 244.5 million patients in China [2]. Chinese guidelines for prevention of CVD are based on hypertension due to the risk stratification to select the best strategies.

Risk stratification based on traditional risk factors was widely used for CVD predicting, such as the model of 30-year risk of CVD in the Framingham study[3], the 10-year risk of CVD in Europe[4] and 10-year risk of CVD in China.[5] But most of adults are classified as a low or intermediate risk by those risk prediction schemes even using lifetime risk estimate, and many of the CVD events occur in the "low" and "intermediate" risk groups[6-8]. This likely results in decreased recognition of risk factors and suboptimal utilization of preventive strategies. The Cardiovascular risk scores do not include markers of organ damage,previous studies proved that markers of organ damage to cardiovascular risk scores may improve the risk assessment for CVD[9-11]. 
Carotid plaque is considered as the marker of early atherosclerosis. It plays a fundamental part in the occurrence of CVD [12-17] and was recommended for evaluation of target of organ damage among patients with hypertension by hypertension guidelines. Patients who had hypertension combined with carotid plaque was classified as a high risk group $[18,19]$. While, about $50 \%$ of patients with hypertension were examined for carotid plaques [20], which was more likely to overestimated the CVD risk.

B-mode ultrasound of carotid arteries was recommended for cardiovascular risk assessment in asymptomatic adults at intermediate risk by the American Heart Association[21]. While carotid arteries is seldom combined risk stratification assessing the risk of CVD. Therefore, we conducted this crosssectional study for evaluating the prevalence of carotid plaque, and the value of risk stratification plus carotid plaque improving the predicting of CVD.

\section{Methods}

\section{Participants}

This cross-sectional survey was conducted from June 2016 to June 2017. We prospectively recruited patients with hypertension who visited clinics. Each participant was required to complete an interviewerled questionnaire and performed carotid artery ultrasound examinations in Anzhen Hospital, Beijing, China. The inclusion criteria were as follows: (1) patients aged 35-74 years; and (2) patients with a medical history of hypertension for a minimum of 3 months. The exclusion criteria were as follows: (1) patients with familial hypercholesterolemia; and (2) patients suffering from serious congestive heart failure, renal insufficiency and requiring dialysis treatment, and/or cancer patients. In total, 4500 subjects met the selection criteria, and 3998 subjects completed traditional risk factors survey and carotid ultrasound measurements[22]. Written informed consent was obtained from participants.

\section{Data collection}

The questionnaire concerned demographic information, medical history, and details of lifestyle. The information was collected by trained research staff. Height, weight, waist circumference and blood pressure (BP) were measured. Systolic BP (SBP) and diastolic BP (DBP) were measured using a standard mercury sphygmomanometer cuff placed on the right arm, supported at the level of the heart.

Participants rested for at least $5 \mathrm{~min}$ in a seated position, then BP was measured twice, with a $60 \mathrm{~s}$ interval, and the mean of these two measurements was recorded and averaged[17, 18]. Total cholesterol (TC) and low-density lipoprotein-cholesterol (LDL-C) during the last three months were recorded in the questionnaire, or were measured if records could not be provided.

Color Doppler ultrasonography was used for carotid artery plaque. The carotid ultrasound scanning and measuring protocol was carried out using a previously described method[20,23]. The distance between the lumen-intima and the media-adventitia ultrasound interface was measured using Hitachi Preirus Color Doppler ultrasound machine with linear array probe (at a frequency of $3 \sim 7 \mathrm{MHz}$ ). All subjects were scanned at six carotid sites [including $1 \mathrm{~cm}$ length of both side of the common carotid artery (CCA), the 
carotid artery bulb (bulb) and the proximal internal carotid artery (ICA)]. 12 sites were visited and measured for CCA, bulb and ICA (three sites within $1 \mathrm{~cm}$ for the far and the near wall of each carotid artery was measured). Intima-media thickness (IMT) was an average of 12 measurements. Threedimensional ultrasound imaging system is Vasqscan system developed by University of Western Ontario. All operators had good experience in ultrasound scanning and measurement, and high-quality data were collected. The result was valid if subjects performed carotid artery ultrasound examinations in Anzhen Hospital within the last year.

\section{Measurement and diagnostic criteria}

Current smokers were defined as those who reported having smoked $\geq 100$ cigarettes during their lifetime and were smoking every day or some days at the time of interview. Obesity was defined as a waist circumference $\geq 80 \mathrm{~cm}$ for women and $\geq 85 \mathrm{~cm}$ for men. Ischemic cardiovascular disease (ISCVD) includes ischemic stroke and coronary heart disease (CHD). A history of ischemic stroke was defined as a history of symptoms/signs such as numbness, abnormal speech, transient blindness, vertigo, nausea, deviated eyes and mouth, hemiplegia, or dribbling; consistent signs on brain computed tomography or magnetic resonance imaging; or the diagnosis of ischemic stroke by a neurologist (including cerebral thrombosis or lacunar infarction). A clinical diagnosis of CHD based on patient symptoms, electrocardiogram changes, and highly sensitive biochemical markers, as well as information gleaned from various imaging techniques, which includes history of stable angina, unstable angina and myocardial infarction. Controlled hypertension was defined as a mean SBP $<140 \mathrm{mmHg}$ and a mean $\mathrm{DBP}<90 \mathrm{mmHg}$. Cardiovascular risk score was calculated using Chinese Risk Tables, which estimate the 10 -year risk of suffering CVD. The risk score for each subject classified into $0-5 \%$ risk (low risk), 5-10\% risk (intermediate risk), $\geq 10 \%$ risk (high risk) [5]. A carotid plaque was defined as carotid IMT ( $\geq 1.5 \mathrm{~mm}$, or thicker than the normal IMT value by $0.5 \mathrm{~mm}$ or thicker than the normal IMT value by $50 \%[20,23]$.

\section{Statistical analysis}

Non-normally distributed variable (duration of hypertension) was presented as median (interquartile range). Normally distributed continuous variables are presented as means \pm standard deviations, and independent two-sample t-test was used to compare the difference between two means. Categorical variables are presented as frequencies and percentages, and the chi-square test were used to compare differences in proportions between groups. All analyses were performed by SPSS statistical software (Version18.0, SPSS Inc., Chicago). P values of $<0.05$ were considered statistically significant.

\section{Results}

\section{General information}

A total of 3,998 patients with hypertension were recruited. The mean age was 57.8 years (range 39-74 years, SD = 8.2), and 32.9\% (1315) were male. The median duration of hypertension was 6 years. There were $2113(52.9 \%)$ subjects who had ideal blood pressure control. The prevalence of CHD and ischemic 
stroke was $4.7 \%$ and $8.8 \%$, respectively. Among patients free from ISCVD $(n=3479)$, there were 884 patients $(25.4 \%)$ at high risk for CVD, and 868 (25.0\%), 1727 (49.6\%) was classified as intermediate risk or low risk. The general characteristics of subjects are listed in Table 1.

Table 1

General characteristics and risk stratification of subjects

\begin{tabular}{|ll}
\hline Characteristics & M (SD)/ n (\%) \\
\hline Male sex, $\mathrm{n}(\%)$ & $1315(32.9)$ \\
\hline Age (y), M (SD) & $57.6 \pm 8.1$ \\
\hline Duration of hypertension (y), $\left.\mathrm{M} \mathrm{Q}_{1}, \mathrm{Q}_{3}\right)$ & $6(3,10)$ \\
\hline Risk factors for risk stratification & \\
\hline SBP (mmHg), M (SD) & $134 \pm 18$ \\
\hline DBP (mmHg), M (SD) & $81 \pm 11$ \\
\hline LDL-C (mmol/L), M (SD) & $3.07 \pm 0.88$ \\
\hline BMI, M (SD) & $26.8 \pm 3.7$ \\
\hline Current smoking, $\mathrm{n}(\%)$ & $839(21.0)$ \\
\hline History of diabetes, $\mathrm{n}(\%)$ & $997(24.9)$ \\
\hline History of ISCVD, $\mathrm{n}(\%)$ & $519(13.0)$ \\
\hline History of CHD & $186(4.7)$ \\
\hline History of ischemic stroke & $351(8.8)$ \\
\hline Risk stratification, $\mathrm{n}(\%)$ & $884(25.4)$ \\
\hline High-risk group & $868(25.0)$ \\
\hline Intermediate-risk group & $1727(49.6)$ \\
\hline Low-risk group & $2113(52.9)$ \\
\hline Hypertension control & \\
\hline Data are & \\
\hline
\end{tabular}

Data are presented as mean \pm standard deviation, median (interquartile range) or $n$ (\%); SBP, Systolic blood pressure; DBP, diastolic blood pressure; LDL-C, low density lipoprotein cholesterol; BMI,body mass index; ISCVD, ischemic cardiovascular disease; CHD, coronary heart disease

\section{Presence of carotid plaques}

A total of 2010 of 3998 patients (50.3\%) were detected for carotid plaques, which were more frequent in men than in women $(P<0.001)$, and increased with age and duration of hypertension (all $P<0.001)$. The 
additional three risk factors were significantly correlated with carotid plaques among controlled hypertension: current smoking, central obesity, and diabetes. The risk factors among controlled hypertension were not consistent with uncontrolled hypertension. The prevalence rate of carotid plaques did not vary by LDL-C group among controlled hypertension, but lower LDL-C concentration were correlated with a significantly decreased risk of carotid plaques among uncontrolled hypertension (Table 2). 
Table 2

Presence of carotid plaques

\begin{tabular}{|c|c|c|c|c|c|c|}
\hline \multirow[t]{2}{*}{ Main characteristics } & \multicolumn{2}{|l|}{ All patients } & \multicolumn{2}{|c|}{$\begin{array}{l}\text { Controlled } \\
\text { hypertension }\end{array}$} & \multicolumn{2}{|c|}{$\begin{array}{l}\text { Uncontrolled } \\
\text { hypertension }\end{array}$} \\
\hline & $\mathrm{n}(\%)$ & $\begin{array}{l}\mathrm{P} \text { - } \\
\text { value }\end{array}$ & $\mathrm{n}(\%)$ & P-value & $\mathrm{n}(\%)$ & P-value \\
\hline Sex & & $<.001$ & & $<0.001$ & & $<0.001$ \\
\hline Female & 1183(44.1) & & $644(42.2)$ & & $539(46.6)$ & \\
\hline Male & $827(62.9)$ & & $384(65.4)$ & & $443(60.9)$ & \\
\hline Age group (y) & & $<.001$ & & $<0.001$ & & $<0.001$ \\
\hline$<45$ & $26(13.1)$ & & $10(9.1)$ & & $16(18.2)$ & \\
\hline $45-54$ & $404(32.1)$ & & $200(29.7)$ & & $204(35.0)$ & \\
\hline $55-64$ & $900(56.2)$ & & $475(55.6)$ & & $425(56.9)$ & \\
\hline$\geq 65$ & $680(72.2)$ & & $343(72.2)$ & & $337(72.2)$ & \\
\hline $\begin{array}{l}\text { Duration of hypertension } \\
\text { (y) }\end{array}$ & & $<0.001$ & & $<0.001$ & & $<0.001$ \\
\hline$<5$ & $824(42.2)$ & & $471(42.2)$ & & $353(42.3)$ & \\
\hline 5- & $556(52.5)$ & & $271(50.7)$ & & $285(54.3)$ & \\
\hline $10-$ & $630(69.8)$ & & $286(61.9)$ & & $344(65.5)$ & \\
\hline Current smoking & & $<001$ & & $<0.001$ & & 0.019 \\
\hline No & 1519(48.1) & & $814(46.2)$ & & 705 (50.5) & \\
\hline Yes & 491(58.5) & & $214(61.1)$ & & 277 (56.6) & \\
\hline Central obesity & & 0.076 & & 0.022 & & 0.589 \\
\hline No & $363(47.4)$ & & $227(44.2)$ & & $136(53.8)$ & \\
\hline Yes & 1647(51.0) & & $801(50.1)$ & & $846(51.8)$ & \\
\hline Diabetes & & 0.001 & & $<0.001$ & & 0.917 \\
\hline No & 1462(48.7) & & $730(45.8)$ & & $732(52.0)$ & \\
\hline Yes & $548(55.0)$ & & $298(57.4)$ & & $250(52.3)$ & \\
\hline LDL-C (mmol/L) & & 0.004 & & 0.278 & & 0.009 \\
\hline
\end{tabular}




\begin{tabular}{|c|c|c|c|c|c|c|}
\hline \multirow[t]{2}{*}{ Main characteristics } & \multicolumn{2}{|l|}{ All patients } & \multicolumn{2}{|c|}{$\begin{array}{l}\text { Controlled } \\
\text { hypertension }\end{array}$} & \multicolumn{2}{|c|}{$\begin{array}{l}\text { Uncontrolled } \\
\text { hypertension }\end{array}$} \\
\hline & $\mathrm{n}(\%)$ & $\begin{array}{l}\mathrm{P}- \\
\text { value }\end{array}$ & $\mathrm{n}(\%)$ & P-value & n (\%) & P-value \\
\hline$<1.8$ & $120(44.4)$ & & $65(42.8)$ & & $55(46.6)$ & \\
\hline $1.8-2.59$ & $439(46.8)$ & & $242(47.0)$ & & $197(46.6)$ & \\
\hline $2.6-3.39$ & $470(49.8)$ & & $263(48.9)$ & & 207 (51.1) & \\
\hline$\geq 3.4$ & $981(53.1)$ & & $458(50.4)$ & & $523(55.1)$ & \\
\hline
\end{tabular}

\section{The relationship between risk stratification and carotid plaques}

Among patients free from ISCVD $(n=3479)$, the detected rate of carotid plaque was $48.5 \%(1689 / 3479)$, and it was $64.7 \%$ (572/884), 53.7\% (466/868), and 38.5\% (651/1727) among patients at high risk to low risk, respectively. While among patients with carotid plaque, a high percentage of patients $(651 / 1689)$ at a low risk were found (Table 3 ).

Table 3

Relationship between risk stratification and carotid plaques among patients free from

ISCVD

\begin{tabular}{|c|c|c|c|}
\hline \multirow[t]{2}{*}{ Risk stratification } & \multicolumn{2}{|c|}{ Carotid plaque } & \multirow[t]{2}{*}{ Total } \\
\hline & Yes & No & \\
\hline High risk & 575 & 312 & 884 \\
\hline Intermediate risk & 466 & 402 & 868 \\
\hline Low risk & 651 & 1076 & 1727 \\
\hline Total & 1689 & 1790 & 3479 \\
\hline
\end{tabular}

\section{Risk stratification plus carotid plaque improving the predicting of ISCVD and reclassification}

A total of $11.8 \%$ and $4.9 \%$ with carotid plaques suffered an ischemic stroke and $\mathrm{CHD}$, and $5.8 \%$ and $4.4 \%$ of patients without carotid plaque suffered an ischemic stroke and CHD, respectively. Thus, carotid plaques were significantly associated with ischemic stroke $(P<0.001)$, and negatively correlated with CHD $(P=0.500)$; the similar result was also obtained in the relationship between risk stratification and ISCVD. The subjects were classified to six groups adding carotid plaque to risk stratification, the combination was significantly correlated to ischemic stroke $(P<0.001)$ and had a more significant effect 
in each risk stratification. The ischemic stroke prevalence increased from 5.3-9.1\% in the low risk group $(P=0.001), 5.4-12.3 \%$ in the intermediate risk group $(P<0.001)$ and $8.2-14.4 \%$ than in the high-risk group $(P=0.004)$. According to the prevalence of ischemic stroke and the results of two groups' comparison, adding carotid plaque to risk stratification resulted in the reclassification. Intermediate risk plus carotid plaque (443/3998) were reclassified to a new high-risk group, high risk only (749/3998) and low risk plus carotid plaque (353/3998) were reclassified to a new intermediate risk group; and intermediate risk only (553/3998) were reclassified to a new low risk group. Adding carotid plaque to risk stratification was not associated with a higher prevalence of $\mathrm{CHD}(\mathrm{P}=0.852)$, increase in CHD prevalence was not significant in each risk stratification (Table 4). According to the reclassification, there were 1635 subjects (40.9\%) at high risk group, and 1102 (27.6\%), 1261 (31.5\%) was classified as intermediate risk or low risk group (Table 5).

Table 4

Relationship between risk stratification only or combined carotid plaque and ISCVD

\begin{tabular}{|c|c|c|c|c|}
\hline \multirow[t]{2}{*}{ Predictors } & \multicolumn{2}{|c|}{ Coronary heart disease } & \multicolumn{2}{|c|}{ Ischemic stroke } \\
\hline & $\mathrm{n}(\%)$ & P-value & $\mathrm{n}(\%)$ & P-value \\
\hline Carotid plaque & \multicolumn{3}{|c|}{0.500} & $<0.001$ \\
\hline Yes $(n=2010)$ & \multicolumn{2}{|l|}{$98(4.9)$} & \multicolumn{2}{|l|}{$238(11.8)$} \\
\hline No $(n=1998)$ & \multicolumn{2}{|l|}{$88(4.4)$} & \multicolumn{2}{|l|}{$116(5.8)$} \\
\hline \multicolumn{2}{|l|}{ Risk stratification } & \multicolumn{2}{|l|}{0.911} & $<0.001$ \\
\hline High risk ( $\mathrm{n}=1061)$ & \multicolumn{2}{|l|}{$51(4.8)$} & \multicolumn{2}{|l|}{$131(12.3)$} \\
\hline Intermediate risk $(\mathrm{n}=996)$ & \multicolumn{2}{|l|}{$44(4.4)$} & \multicolumn{2}{|l|}{$92(9.2)$} \\
\hline Low risk $(n=1941)$ & \multicolumn{2}{|l|}{$91(4.7)$} & \multicolumn{2}{|l|}{$131(6.7)$} \\
\hline \multicolumn{2}{|l|}{ Risk stratification plus carotid plaque } & \multicolumn{2}{|l|}{0.852} & $<0.001$ \\
\hline High risk + carotid plaque $(n=1192)$ & \multicolumn{2}{|l|}{$38(5.4)$} & $102(14.4)$ & 0.004 \\
\hline High risk only $(n=749)$ & $13(3.7)$ & & \multicolumn{2}{|l|}{$29(8.2)$} \\
\hline Intermediate risk + carotid plaque $(n=443)$ & \multicolumn{2}{|l|}{$26(4.7)$} & $68(12.3)$ & $<0.001$ \\
\hline Intermediate risk only $(n=553)$ & $18(4.1)$ & & \multicolumn{2}{|l|}{$24(5.4)$} \\
\hline Low risk + carotid plaque $(n=353)$ & \multicolumn{2}{|l|}{$34(4.5)$} & $68(9.1)$ & 0.001 \\
\hline Low risk only $(n=708)$ & \multicolumn{2}{|l|}{$57(4.8)$} & \multicolumn{2}{|l|}{$63(5.3)$} \\
\hline \multicolumn{5}{|c|}{$\begin{array}{l}\text { No significance between high risk plus carotid plaque and intermediate risk plus carotid plaque }(P= \\
0.276) \text {; }\end{array}$} \\
\hline \multicolumn{5}{|c|}{ No significance between high risk only and low risk plus carotid plaque $(P=0.637)$; } \\
\hline \multicolumn{5}{|c|}{ No significance between low risk only and intermediate risk $(P=0.916)$} \\
\hline
\end{tabular}


Table 5

Reclassification to risk stratification of ISCVD

\begin{tabular}{|llll|}
\hline Group of reclassification & Presentation & N & $\%$ \\
\hline New-High risk & High risk + plaque & 1635 & 40.9 \\
\hline & Intermediate risk + plaque & & \\
\hline New-Intermediate risk & High risk only & 1102 & 27.6 \\
\hline New-Low risk & Low risk + plaque & & \\
\hline & Intermediate risk only & 1261 & 31.5 \\
\hline & Low risk only & & \\
\hline
\end{tabular}

\section{Discussion}

Our study indicated that more than half of the patients with hypertension were detected carotid plaque. Carotid plaque and risk stratification did not appear to be correlated with CHD, but closely correlated with ischemic stroke. Risk stratification plus carotid plaque was superior to carotid plaque or risk stratification alone for assessing the risk of ischemic stroke.

Most previous studies proved carotid IMT and carotid plaque are strong predictors of future stroke, myocardial infarction and vascular death, and the presence of carotid plaques was superior to carotid IMT for assessing the risk of CVD [12-17]. Few studies did not support this conclusion. Naylor concluded that a very large majority of asymptomatic carotid artery stenosis patients will never develop a stroke, only $1 \%$ of strokes will be prevented by a mass campaign of intervention, and $94 \%$ of interventions in asymptomatic carotid artery stenosis patients may be unnecessary[24]. The current study showed, carotid plaque did not appear to be correlated with CHD, but closely correlated with ischemic stroke.

CVD risk prediction models based on traditional risk factors have formed the basis for the CVD primary prevention, but their usefulness as predictors is also limited as many of the CHD events occur in the "low" and "intermediate" risk groups even using lifetime risk estimate [6-8]. Current risk charts do not include organ damage markers, which was recommended to be used in cardiovascular risk stratification by current hypertension guidelines rather than other risk factors such as familial history of early cardiovascular events and serum uric acid $[18,19]$. Several reports demonstrated that markers of cardiac, vascular and renal organ damage have been associated to relatively high sensibility or specificity and independent predictive value of high risk profile, and the combination of risk score and organ damage may improve cardiovascular risk prediction, but did not formed a reclassification chart[9-11]. Nambi V, et al [25] proved carotid IMT and the presence or absence of plaque improved CHD risk prediction. Adding carotid IMT and plaque information to traditional risk factors resulted in the reclassification of $23 \%$ of the subjects. Almost $61.9 \%$ of those reclassified from the intermediate risk group were reclassified to a lower 
risk. Furthermore, nobody from the low-risk group was reclassified to a high-risk group, and nobody from the high-risk group was reclassified to the low-risk group. In the current study, adding carotid plaque had a more significant effect on ischemic stroke than resulted in the reclassification. Intermediate risk plus carotid plaque was reclassified to a high-risk group, low risk plus carotid plaque was reclassified to an intermediate risk group, while high risk only and intermediate risk only were reclassified to a lower risk. But the correlation to CHD was not increased significantly.

Our study showed that $25.4 \%$ of patients with hypertension were classified as high-risk group according to traditional risk prediction schemes, and at least $50.3 \%$ of patients who had hypertension coupled with carotid plaques were considered as high-risk group according to hypertension guidelines. There was a big difference between the two approaches. Adding carotid plaque to traditional risk prediction schemes, the new risk stratification seemed to have a more reasonable outcome with $40.9 \%$ of high-risk group. Although the result needs to be confirmed by large sample observational studies, it provides insights for improving traditional cardiovascular risk assessment.

The effective approach to restrict the health burden is to prevent CVD from developing at the earliest stage of atherosclerosis. Thus, screenings for patients at a high risk or patients with carotid plaque are needed. Consistent with other previous studies, the carotid plaque detection rate increased significantly with age, and the traditional cardiovascular risk factors also contributed to the development of carotid atherosclerosis[20]. There was ample evidence that lifestyle changes coupled with medication to control blood pressure and blood sugar or lower cholesterol appeared to slow the progression of carotid IMT [26-28].

The present study has several limitations. Firstly, traditional risk stratification plus carotid plaque resulted in the reclassification for CVD assessing, it is useful because it can be used simply in clinic, given the cross-sectional study, advanced observational study should be done to test the ability for predicting CVD as a new assessment tools. Secondly, cardiovascular risk stratification was based on Chinese risk prediction models and score chart, lifetime risk estimate did not conducted. Thus, the result provided another approach improving cardiovascular risk stratification. Finally, a small size of samples from young patients enrolled in this study, which may increase the detection of carotid plaques among patients with hypertension.

\section{Conclusions}

In this study, half of the patients with hypertension were detected carotid plaque, and carotid plaque did not appear to be correlated with CHD, but closely correlated with ischemic stroke. One quarter was grouped into high risk according to Chinese CVD risk chart, the combination of carotid plaque and risk stratification chart may improve risk prediction of CVD and provided a new risk stratification chart, it may better identify individual risk and help physicians in both therapeutic and preventive strategies.

\section{Abbreviations}


CVD: Cardiovascular disease

BP: blood pressure

SBP: Systolic BP

DBP: Diastolic BP

TC: Total cholesterol

LDL-C: Low-density lipoprotein-cholesterol

CCA: Common carotid artery

ICA: Internal carotid artery

ISCVD: Ischemic cardiovascular disease

CHD: Coronary heart disease

\section{Declarations}

\section{Ethics approval and consent to participate}

This study was conducted according to the recommendations of the Declaration of Helsinki and was approved by the institutional review boards of the sixth clinical institute, Capital Medical University, Beijing, China. Written consent was obtained from all participants.

\section{Consent for publication}

Not applicable.

\section{Availability of data and materials}

The raw dataset analyzed in the current study are available from the corresponding author on reasonable request.

\section{Competing interests}

The authors declare that they have no competing interests.

\section{Funding}

This study was funded by the Beijing Municipal Health Commission, China (TG-2015-33)

\section{Authors' contributions}


ZHJ and LJ conceived and designed the study. ZHJ, WJW and YHX collected and analysed data. LJ and SXT contributed to interpreted results. ZHJ and SXT wrote the manuscript. All authors read and approved the final manuscript.

\section{Acknowledgements}

We thank all the experienced operators for ultrasound scanning and measurement which led to high quality results.

\section{Conflict of interests}

The authors declare that they have no conflict of interest.

\section{References}

1. Yusuf PS, Joseph P, Rangarajan S, Islam S, Mente A, Hystad P, et al. Modifiable risk factors, cardiovascular disease, and mortality in 155722 individuals from 21 high-income, middle-income, and low-income countries (PURE): a prospective cohort study. 2020; 395:795-808.

2. Wang Z, Chen Z, Zhang L, Wang X, Hao G, Zhang Z, et al. Status of hypertension in China: Results of hypertension survey, 2012-2015. Circulation. 2018;137:2344-2356.

3. Pencina MJ, D'Agostino RB, Larson MG, Massaro JM, Vasan RS. Predicting the 30-year risk of cardiovascular disease: the Framingham heart study. Circulation. 2009;119:3078-3084.

4. Piepoli MF, Hoes AW, Agewall S, Albus C, Brotons C, Catapano AC, et al. 2016 European Guidelines on cardiovascular disease prevention in clinical practice: The Sixth Joint Task Force of the European Society of Cardiology and Other Societies on Cardiovascular Disease Prevention in Clinical Practice (constituted by representatives of 10 societies and by invited experts) Developed with the special contribution of the European Association for Cardiovascular Prevention \& Rehabilitation (EACPR). Eur Heart J. 2016; 37: 2315-2381.

5. Wu YF, Liu XP, Li X, Li Y, Zhao L, Chen Z, et al. Estimation of 10-year risk of fatal and non fatal ischemic cardiovascular disease in Chinese adult. Circulation. 2006; 114:2217-2223.

6. Gooding HC, Ning H, Gillman MW, Shay C, Allen N, Goff DC, et al. Application of a Lifestyle-Based Tool to Estimate Premature Cardiovascular Disease Events in Young Adults: The Coronary Artery Risk Development in Young Adults (CARDIA) Study. JAMA Intern Med. 2017; 177:1354-1360.

7. Schlendorf KH, Nasir K, Blumenthal RS. Limitations of the Framingham risk score are now much clearer. Prev Med. 2009;48:115-116.

8. Redon J. Global cardiovascular risk assessment: strengths and limitations. High Blood Press Cardiovasc Prev. 2016; 23:87-90.

9. Sehestedt T, Jeppesen J, Hansen TW, Wachtell K, Ibsen H, Torp-Pedersen C, et al. Risk prediction is improved by adding markers of subclinical organ damage to SCORE. Eur Heart J. 2010;31:883-891 
10. Volpe M, Battistoni A, Tocci G, Rosei EA, Catapano AL, Coppo R, et al. Cardiovascular risk assessment beyond Systemic Coronary Risk Estimation: a role for organ damage markers. Hypertens. 2012;30:1056-1064.

11. Tocci G, Figliuzzi I, Presta V, Attalla El Halabieh N, Citoni B, Coluccia R, et al. Adding markers of organ damage to risk score models improves cardiovascular risk assessment: Prospective analysis of a large cohort of adult outpatients - ScienceDirect. International Journal of Cardiology. 2017; 248:342348.

12. Johnsen SH, Mathiesen EB. Carotid plaque compared with intima-media thickness as a predictor of coronary and cerebrovascular disease. Current cardiology Report. 2009;11:21-27.

13. Johnson HM, Stein JH. Measurement of carotid intima-media thickness and carotid plaque detection for cardiovascular risk assessment. Journal of Nuclear Cardiology. 2011; 18:153-162.

14. Van den Oord SC, Sijbrands EJ, Ten Kate GL, Van klaveren D, Van Domburg RT, Van der Ste, et al. Carotid intima-media thickness for cardiovascular risk assessment: systematic review and metaanalysis. Atherosclerosis. 2013;228: 1-11.

15. Joseph FP, Daniel HO'L. Carotid Intima-Media Thickness as Surrogate for and Predictor of CVD. Heart. 2016;11:295-312.

16. Li W, Ma F, Jiang Y, Li JJ, Wu SL. Association between carotid artery plaques and all-cause mortality and cardiovascular events. Zhonghua Xin Xue Guan Bing Za Zhi. 2017;45:1068-1090.

17. Saba L, Saam T, Jäger HR, Yuan C, Hatsukami TS, Saloner D, et al. Imaging biomarkers of vulnerable carotid plaques for stroke risk prediction and their potential clinical implications. Lancet Neurol.2019;18:559-572.

18. The task Force for the Management of Arterial Hypertension of the European Society of Cardiology (ESC) and the European Society of Hypertension (ESH). 2018 ESC/ESH Guidelines for management of arterial hypertension. Eur Heart J. 2018;39:3021-3104.

19. Joint Committee for Guideline Revision. 2018 Chinese Guidelines for Prevention and Treatment of Hypertension-A report of the Revision Committee of Chinese Guidelines for Prevention and Treatment of Hypertension. Journal of Geriatric Cardiology. 2019; 16: 182-241

20. Zuo HJ, Song XT, Wang JW, Deng LQ. A risk score for carotid plaque as an assessment risk of cardiovascular risk among patients with hypertension. Journal of the American Society of Hypertension. 2018;12:833-840.

21. Greenland P, Alpert JS, Beller GA, Benjamin EJ, Budoff MJ, Fayad ZA, et al. 2010 ACCF/AHA guideline for assessment of cardiovascular risk in asymptomatic adults a report of the American college of cardiology foundation/ American heart association task force on practice guidelines. J Am Coll Cardiol. 2010;56: e 50-103.

22. Zuo HJ, Song XT, Wang JW, Deng LQ. A risk score for carotid plaque as an assessment risk of cardiovascular risk among patients with hypertension. Journal of the American Society of Hypertension. 2018;12:833-840. 
23. Yand Y, Huo Y. Standardization of ultrasound Procedure in carotid atherosclerosis: the carotid ultrasound scanning and measuring protocol in the project of establishment of an integrated system for CHD prevention and treatment. Chin J Intervent Cardio. 2008; 16:337-338.

24. Schneider PA, Naylor AR. Transatlantic debate. Asymptomatic carotid artery stenosis-medical therapy alone versus medical therapy plus carotid endarterectomy or stenting. Eur J Vasc Endovasc Surg. 2010; 40:274-281.

25. Nambi V, Chambless L, Folsom AR, He M, Hu Y, Mosle T, et al. Carotid Intima-Media Thickness and Presence or Absence of Plaque Improves Prediction of Coronary Heart Disease Risk: The ARIC (Atherosclerosis Risk in Communities) Study. J Am Coll Cardiol. 2010;55:1600-1607.

26. Whayne, Thomas. Assessment of Carotid Artery Stenosis and the Use of Statins. International Journal of Angiology. 2015; 24:173-178.

27. Tripolt NJ, Narath SH, Eder M, Pieber TR, Wascher TC, Sourij H. Multiple risk factor intervention reduces carotid atherosclerosis in patients with type 2 diabetes. Cardiovasc Diabetol. 2014;13:95.

28. Messas E, Goudot G, Halliday A, Sitruk J, Mirault T, Khider L, et al. Management of carotid stenosis for primary and secondary prevention of stroke: state-of-the-art 2020: a critical review. Eur Heart J Suppl. 2020;22(Suppl M):M35-M42. 